EPJ Web of Conferences 59, 13019 (2013)

DOI: $10.1051 /$ epjconf/20135913019

(C) Owned by the authors, published by EDP Sciences, 2013

\title{
In situ calibration of the Gamma Reaction History instrument using reference samples ("pucks") for areal density measurements
}

\author{
N.M. Hoffman ${ }^{1}$, H.W. Herrmann ${ }^{1}$, Y.H. Kim¹ ${ }^{1}$, H.H. Hsu ${ }^{1}$, C.J. Horsfield ${ }^{2}$, \\ M.S. Rubery ${ }^{2}$, D.C. Wilson ${ }^{1}$, W. Stoeffl ${ }^{3}$, C.S. Young ${ }^{1}$, J.M. Mack ${ }^{1}$, \\ E.K. Miller ${ }^{4}$, E. Grafil ${ }^{5}$, S.C. Evans ${ }^{1}$, T.J. Sedillo ${ }^{1}$, V.Yu. Glebov ${ }^{6}$ \\ and T. Duffy ${ }^{6}$
}

1 Los Alamos National Laboratory, PO Box 1663, Los Alamos, New Mexico 87545, USA

2 Atomic Weapons Establishment, Aldermaston, Berkshire RG7 4PR, UK

${ }^{3}$ Lawrence Livermore National Laboratory, Livermore, California 94550, USA

4 National Security Technologies, LLC, Santa Barbara, California 93111, USA

${ }^{5}$ Colorado School of Mines, Golden, Colorado 80401, USA

${ }^{6}$ Laboratory for Laser Energetics, University of Rochester, Rochester, New York 14623, USA

\begin{abstract}
The introduction of a sample of carbon, for example a disk or "puck", near an imploding DTfilled capsule creates a source of ${ }^{12} \mathrm{C}$ gamma rays that can serve as a reference for calibrating the response of the Gamma Reaction History (GRH) detector [1]. Such calibration is important in the measurement of ablator areal density $\langle\rho R\rangle_{a b l}$ in plastic-ablator DT-filled capsules at OMEGA [2], by allowing $\langle\rho R\rangle_{a b l}$ to be inferred as a function of ratios of signals rather than from absolute measurements of signal magnitudes. Systematic uncertainties in signal measurements and detector responses therefore cancel, permitting more accurate measurements of $\langle\rho R\rangle_{a b l}$.
\end{abstract}

\section{PUCK GAMMA RAYS}

Materials placed near the intense source of 14.1-MeV neutrons emanating from an imploded fusion capsule can produce gamma rays resulting from the neutrons' inelastic scattering and nuclear capture. To be useful as a reference, a sample disk or "puck" of material must have well characterized mass and dimensions, be neutronically thin, and be located at a known distance from the capsule. Given the measured DT neutron yield $Y_{n D T}$ from the capsule, and the solid angle $\Delta \Omega_{\text {puck }}$ subtended by the puck at the capsule's centre, the total gamma-ray yield of a graphite puck arising from inelastic neutron scattering and captures on ${ }^{12} \mathrm{C}$ is

$$
Y_{\gamma C, p u c k}=\frac{\sigma_{n C}}{m_{C}}\left\langle\rho_{C} R\right\rangle_{p u c k} Y_{n D T} \frac{\Delta \Omega_{\text {puck }}}{4 \pi},
$$

where the areal density of the puck is $\left\langle\rho_{C} R\right\rangle_{\text {puck }}$ and $\sigma_{n C}$ is the total gamma-ray production cross-section of carbon for DT neutrons at $14.1 \mathrm{MeV}$, summed over all nuclear levels and processes, indexed by $k$ :

$$
\sigma_{n C} \equiv \sum_{k} \sigma_{n C k} .
$$

The expression for $Y_{\gamma C \text {,puck }}$ is derived based on picturing the puck as a small segment of a complete spherical shell centred on the capsule. For a thin circular disk of diameter $d$, thickness

This is an Open Access article distributed under the terms of the Creative Commons Attribution License 2.0, which permits unrestricted use, distribution, and reproduction in any medium, provided the original work is properly cited. 
$T$, density $\rho$, located at a distance $D$ from the capsule's centre, $\left\langle\rho_{C} R\right\rangle_{p u c k}=\rho T$ and $\Delta \Omega_{\text {puck }} / 4 \pi=$ $\pi(d / 2)^{2}\left(1 / 4 \pi D^{2}\right)=(d / 4 D)^{2}$. So

$$
\left\langle\rho_{C} R\right\rangle_{p u c k} \frac{\Delta \Omega_{\text {puck }}}{4 \pi}=\rho T \frac{d^{2}}{16 D^{2}}=\frac{M_{\text {puck }}}{4 \pi D^{2}},
$$

where $M_{\text {puck }} \equiv \pi \rho T d^{2} / 4$ is the mass of the puck, and the gamma-ray yield can be written as

$$
Y_{\gamma C, \text { puck }}=\frac{\sigma_{n C}}{m_{C}} Y_{n D T} \frac{M_{\text {puck }}}{4 \pi D^{2}} .
$$

The time-integrated GRH signal arising from the puck when the GRH threshold is $E^{t h r}$ is proportional to its gamma-ray yield:

$$
S_{\text {puck }}=\left\langle R\left(\gamma_{C} ; E^{t h r}\right)\right\rangle Y_{\gamma C, \text { puck }} f_{1}(\theta),
$$

where $\left\langle R\left(\gamma_{C} ; E^{t h r}\right)\right\rangle$ is the mean response of the GRH detector, averaged over the entire ${ }^{12} \mathrm{C}$ gamma-ray spectrum, at threshold $E^{t h r}$. The puck does not emit gamma rays isotropically, because the neutrons irradiating it occupy a narrow beam originating at the capsule, so the expression $f_{1}(\theta)$ accounts for the angular dependence of the puck's emission; $\theta$ is the angle between the direction the neutrons travel from capsule to puck, and the direction from puck to detector. Then using Eq. 1,

$$
S_{\text {puck }}=\left\langle R\left(\gamma_{C} ; E^{t h r}\right)\right\rangle \frac{\sigma_{n C}}{m_{C}} Y_{n D T} \frac{f_{1}(\theta) M_{\text {puck }}}{4 \pi D^{2}},
$$

which may be inverted to solve for the mean response multiplied by $\sigma_{n C} / m_{C}$.

Using the calibrated value of $\left\langle R\left(\gamma_{C} ; E^{t h r}\right)\right\rangle \sigma_{n C} / m_{C}$, the ablator areal density for a plastic-shell capsule can be found from observed signals using the expression [3]

$$
\langle\rho R\rangle_{\text {abl,low }}=\frac{f_{1}(\theta) M_{\text {puck }}}{4 \pi f_{C} D^{2}} \frac{S_{\text {low }}^{\prime}}{S_{\text {puck }, \text { low }}^{\prime}}\left(1-\frac{1}{\mathcal{F}_{D T}} \frac{\left\langle S^{\prime}\right\rangle_{H T S}}{S_{\text {low }}^{\prime}}\right)+\frac{1}{\mathcal{F}_{D T}} \frac{S_{\text {puck,high }}^{\prime}}{S_{\text {puck,low }}^{\prime}}\langle\rho R\rangle_{\text {abl,HTS }} .
$$

$S_{\text {low }}^{\prime}$ is the low-threshold $(\sim 3 \mathrm{MeV})$ signal from which the capsule's areal density is to be inferred, $\left\langle S^{\prime}\right\rangle_{H T S}$ and $\langle\rho R\rangle_{a b l, H T S}$ denote the mean signal and areal density obtained from a reference set of high-threshold $(\sim 5 \mathrm{MeV})$ capsule shots, while $S_{\text {puck,high }}^{\prime}$ and $S_{\text {puck,low }}^{\prime}$ are the puck signals for high and low threshold, and $f_{c} \equiv\left\langle\rho_{C} R\right\rangle /\langle\rho R\rangle_{a b l}$ is the fractional composition by mass of carbon in the $\mathrm{CH}$ ablator. (Signals written as primed quantities have been normalized by neutron yield and photomultiplier quantum efficiency $Q$ and gain $G$, i.e., $S^{\prime} \equiv S / Q G Y_{n D T}$.) $F_{D T}$ is the ratio of mean GRH detector response to DT gamma rays at high and low threshold, averaged over the entire DT gamma-ray spectrum. The form of Eq. 2 explicitly allows for the possibility that ${ }^{12} \mathrm{C}$ gamma rays contribute to the high-threshold GRH signal as well as the low-threshold signal, so that $S_{\text {puck,high }}^{\prime} \neq 0$.

Puck calibration accounts for all processes by which ${ }^{12} \mathrm{C}$ creates gamma rays via neutron inelastic scattering or neutron capture, whether to the $1^{\text {st }}$ excited level or to the $2^{\text {nd }}$ and higher levels, as has been observed in tokamak plasmas [4]. Since the angular dependence of emission from higher levels may differ from $f_{1}(\theta)$, we will field pucks at various values of $\theta$ to measure the effect. Ideally the puck should have the same composition as the ablator, since otherwise we must correct for any differences.

\section{ANGULAR DEPENDENCE OF PUCK GAMMA-RAY EMISSION}

For the function $f_{1}(\theta)$ we use the work of Anderson et al. [5] and Benveniste et al. [6], both of whom expressed the differential cross-section for $\gamma$-ray emission from the 1 st excited level of ${ }^{12} \mathrm{C}$ as

$$
\frac{d \sigma_{n C 1}}{d \Omega}(\theta)=\frac{d \sigma_{n C 1}}{d \Omega}(\pi / 2)\left(1+a \cos ^{2} \theta-b \cos ^{4} \theta\right) .
$$


Table 1. Puck calibration shots. (Tabulated values of $S^{\prime}$ are time integrated from 216 to $217 \mathrm{~ns}$.)

\begin{tabular}{|c|c|c|c|c|c|c|c|}
\hline $\begin{array}{l}\text { OMEGA } \\
\text { shot \# } \\
\text { with } \\
\text { puck }\end{array}$ & $\begin{array}{c}\text { OMEGA } \\
\text { shot \# } \\
\text { without } \\
\text { puck }\end{array}$ & Date & $\begin{array}{c}\text { DT neutron } \\
\text { yield (with puck) } \\
Y_{n D T}\end{array}$ & $\begin{array}{c}\text { PMT } \\
\text { gain } \\
G\end{array}$ & $\begin{array}{c}\text { PMT } \\
\text { quantum } \\
\text { eff } Q\end{array}$ & $\begin{array}{c}\begin{array}{c}\text { Normalized } \\
\text { puck signal }\end{array} \\
S^{\prime} \equiv S / Q G Y_{n D T}\end{array}$ & $\begin{array}{c}\text { Total } \\
\text { uncert in } \\
S^{\prime}(\%)\end{array}$ \\
\hline \multicolumn{8}{|c|}{ 3-MeV ("low threshold") shots } \\
\hline 58165 & 58164 & $5 / 25 / 10$ & $1.60 \mathrm{E} 13$ & 40500 & 0.073 & 6.35E-18 & 10 \\
\hline 61881 & 61887 & $4 / 13 / 11$ & $1.79 \mathrm{E} 13$ & 34771 & 0.084 & 7.07E-18 & 19 \\
\hline 63634 & 63629 & $9 / 27 / 11$ & $3.14 \mathrm{E} 13$ & 34771 & 0.084 & 6.22E-18 & 22 \\
\hline \multicolumn{6}{|c|}{ Average 3-MeV puck signal $\pm R M S \times N /(N-1):$} & \multicolumn{2}{|l|}{$(6.51 \pm 0.84) E-18$} \\
\hline \multicolumn{8}{|c|}{ 5-MeV ("high threshold") shots } \\
\hline 58215 & 58214 & $5 / 27 / 10$ & $8.36 \mathrm{E} 12$ & 40500 & 0.073 & 7.6E-21 & 5100 \\
\hline 63623 & 63626 & $9 / 27 / 11$ & $9.83 \mathrm{E} 13$ & 34771 & 0.084 & 2.7E-19 & 79 \\
\hline 63624 & 63626 & $9 / 27 / 11$ & $7.25 \mathrm{E} 13$ & 34771 & 0.084 & 3.3E-20 & 640 \\
\hline \multicolumn{6}{|c|}{ Average 5-MeV puck signal $\pm R M S \times N /(N-1):$} & \multicolumn{2}{|l|}{$(1.2 \pm 1.4) \mathrm{E}-19$} \\
\hline
\end{tabular}

Then

$$
f_{1}(\theta) \equiv \frac{d \sigma_{n C 1}}{d \Omega}(\theta) /\left\langle\frac{d \sigma_{n C 1}}{d \Omega}\right\rangle
$$

where the denominator is the mean value of the differential cross-section:

$$
\left\langle\frac{d \sigma_{n C 1}}{d \Omega}\right\rangle \equiv \frac{1}{4 \pi} \int_{4 \pi} \frac{d \sigma_{n C 1}}{d \Omega}(\theta) d \Omega=\frac{1}{2} \int_{0}^{\pi} \frac{d \sigma_{n C 1}}{d \Omega}(\theta) \sin \theta d \theta=\frac{d \sigma_{n C 1}}{d \Omega}(\pi / 2)\left(1+\frac{a}{3}-\frac{b}{5}\right) .
$$

So

$$
f_{1}(\theta) \equiv \frac{1+a \cos ^{2} \theta-b \cos ^{4} \theta}{1+a / 3-b / 5} .
$$

For our puck experiments, $\theta=43^{\circ}$, so $\cos \theta=0.731$. Anderson et al. found $a=1.75 \pm 0.18$ and $b=1.20 \pm 0.31$, leading to $f_{1}\left(43^{\circ}\right)=1.19 \pm 0.02$, while Benveniste et al. found $a=3.01 \pm 0.38$ and $b=2.56 \pm 0.40$, giving $f_{1}\left(43^{\circ}\right)=1.26 \pm 0.03$. (The uncertainty in $f_{1}$ comes from evaluating the linear variation of $f_{1}$ with $a$ and $b$.) We use the average of these two values, $f_{1}\left(43^{\circ}\right)=1.23 \pm 0.05$, where the uncertainty represents systematic uncertainty.

\section{PUCK DATA}

We have data from several pairs of similar shots in which one member of the pair had a puck placed near the capsule, while the other member did not. The capsule-only signal furnishes a background record that is subtracted from the capsule+puck signal, leaving the signal from the puck alone, which is then integrated in time. Table 1 shows results for three pairs of such shots done at 3-MeV GRH threshold and three pairs done at 5-MeV threshold.

A fourth pair of 3-MeV shots gave a discrepant result, perhaps owing to the high level of signal noise on the capsule+puck shot, and is not included. Signal traces for the low-threshold pair \#58165/\#58164 are shown in Fig. 1. The mass of the puck for these shots $M_{\text {puck }}=9.92 \mathrm{~g}$ and its distance from the capsule $\mathrm{D}=6.60 \mathrm{~cm}$ were measured quite accurately and with negligible systematic uncertainty.

Random uncertainty in time-integrated signals is assumed to arise from three contributions: electrical oscillations in the time-dependent record, uncertainty in defining the background, and Poisson statistical noise. Since the no-puck signal for each pair furnishes a reliable background, we regard the uncertainty in the background as negligible. The Poisson noise is estimated by determining the yield of gamma 


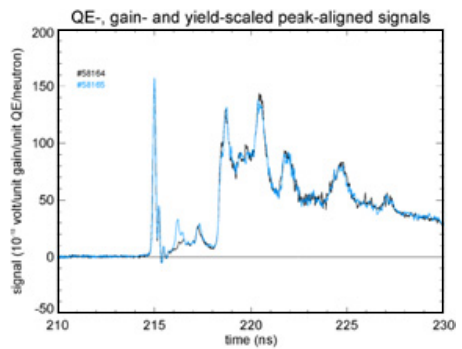

Figure 1. Normalized GRH signals $S^{\prime}(t) \equiv S(t) / Q G Y_{n D T}$ for OMEGA shots \#58164 (black) and \#58165 (light blue), at low threshold ( $3 \mathrm{MeV}$ ). Puck signal on shot \#58165 is evident as a small peak centered at $216.3 \mathrm{~ns}$.

rays from the puck using Eq. 1 with $\sigma_{n C} / m_{C}=\sigma_{n C 1} / m_{C}=0.0112 \mathrm{~cm}^{2} / \mathrm{g}$, using $\sigma_{n C 1}=(0.223 \pm$ $0.009) \times 10^{-24} \mathrm{~cm}^{2}$ obtained from a search of published literature [7]. The gamma ray signal in the GRH takes various forms (gamma rays, Compton electrons, Cherenkov photons, photoelectrons) in transit, so the Poisson noise is determined by the particle whose numbers are the smallest as the signal travels, typically the Compton electrons and photoelectrons [3]. In practice, for puck signals, the Poisson noise contribution of $2-4 \%$ is negligible compared to other sources of uncertainty, the most important of which is electrical oscillation noise, as seen in Fig. 1. To estimate its effect on time-integrated signals, we computed integrals by choosing a fixed lower limit for the range of integration (e.g., $215.5 \mathrm{~ns}$ in Fig. 1) while allowing the upper limit of the integration range to vary over a pre-determined interval, for example, 217 to 219 ns. Performing a large number of such integrals, each with a different upper limit, then furnishes a sample population of values whose standard deviation is an estimate of the uncertainty in the integrated signal. While this method can be biased by a slope in the baseline, in the best cases it leads to an estimate of $\sim 8 \%$ for puck signals, but can be as large as $\sim 20 \%$. In addition to random uncertainty, we include 5\% yield uncertainty, but no uncertainty in PMT gain or quantum efficiency, since yields vary shot-to-shot but the same PMT is generally used on the puck and no-puck shots.

Subtracting the no-puck signal from the capsule+puck signal increases the electrical noise compared to either signal separately. But this effect is reduced by averaging the normalized puck signals over all three pairs. The resulting uncertainty-weighted mean value for $3-\mathrm{MeV}$ measurements is $S_{\text {puck,low }}^{\prime}=(6.51 \pm 0.56) \times 10^{-18} \mathrm{~V}$ ns/unit quantum efficiency/unit gain/neutron. For a large hypothetical population of puck signals, the population's standard deviation $\sigma$ is approximately $N /(N-1)$ times larger than the sample's $\sigma$, for a sample of size $N$. Thus for $N=3$ we estimate the value of the population $\sigma=0.84 \times 10^{-18}$. This value, about $13 \%$ of the mean, is shown in Table 1 .

The puck is probably not detected at $5-\mathrm{MeV}$ threshold, even on shots with clean signals and low noise. To search for the signal, we defined a "template" representing our knowledge of what a puck signal looks like, by averaging the $3-\mathrm{MeV}$ signals and normalizing the result to have unit integrated area. We could then find the scale factor that gave the best fit of the template to the 5-MeV signal, after the no-puck record had been subtracted. This approach gives smaller uncertainties than a straightforward application of the technique used for 3-MeV puck signals. Even so, $S_{\text {puck, high }}^{\prime}$ has large uncertainty, as shown in Table I. (The puck on shot 58215 was at a different $\theta$ than the others, but we ignore that effect here since in practice no puck signal is seen.) Fortunately inferred areal densities are not sensitive to $S_{\text {puck, high }}^{\prime}$, since the ratio $\mathrm{F}_{a b l} \equiv S_{\text {puck,high }}^{\prime} / S_{\text {puck,low }}^{\prime}=6.51 \times 10^{-18} / 1.2 \times 10^{-19}=0.018 \pm 0.021$ is small even though it is uncertain.

\section{References}

[1] H. W. Herrmann et al., J. Phys.: Conf. Ser. 244, 032047 (2010)

[2] T. R. Boehly et al., Opt. Commun. 133, 495 (1997) 


\section{IFSA 2011}

[3] N. M. Hoffman et al., to be submitted to Phys. Plasmas (2012)

[4] V. G. Kiptily, F. E. Cecil, S. S. Medley, Plasma Phys. Control. Fusion 48, R59-R82 (2006)

[5] J. D. Anderson et al., Phys. Rev. 111, 572 (1958)

[6] J. Benveniste, A. C. Mitchell, C. D. Schrader, and J. H. Zenger, Nucl. Phys. 19, 448 (1960)

[7] B. T. S. Barton and J. R. Langenbrunner, LANL document LA-UR-10-04522 (2010) 